\title{
Microflaring of a solar bright point ${ }^{\star}$
}

\author{
O. Vilhu ${ }^{1, \star \star}$, J. Huovelin ${ }^{1}$, S. Pohjolainen ${ }^{2}$, J. Virtanen ${ }^{1}$, and W. Curdt ${ }^{3, \star \star \star}$ \\ 1 Observatory, Box 14, 00014 University of Helsinki, Finland \\ e-mail: osmi.vilhu@helsinki.fi; huovelin@astro.helsinki.fi \\ 2 Tuorla Observatory, University of Turku, Finland \\ e-mail: spo@astro.utu.fi \\ 3 Max-Planck-Institut für Aeronomie, 37191 Katlenburg-Lindau, Germany \\ e-mail: curdt@mpae.gwdg.de
}

Received 24 April 2002 / Accepted 11 July 2002

Abstract. A $50 \times 50$ arcsec region near the solar disc center containing a bright point (BP) was observed with the SUMERspectrograph of the $\mathrm{SOHO}$ observatory. The data consist of two hours observation of four far-UV emission lines formed between $2 \times 10^{4}-6 \times 10^{5} \mathrm{~K}$, with 2 arcsec spatial, 2.8 min temporal and $4 \mathrm{~km} \mathrm{~s}^{-1}$ spectral resolution. A striking feature was the strong microflaring of the major persistent $\mathrm{BP}$ (with size $8 \times 8 \mathrm{arcsec}$ ) and the appearance of several short lived transients. The microflaring of each individual $2 \times 2$ arcsec pixel inside the main BP was coherent, indicating strong interaction of the possible sub arc sec building blocks (magnetic flux tubes). Using the emission measure at $10^{5} \mathrm{~K}$ as an indicator of the loop foot point area and magnetic filling factor, we suggest 10 per cent filling factor for the BP observed. This is similar to that on the average surface of a medium-active solar type star.

Key words. Sun: UV radiation - Sun: chromosphere - Sun: transition region - Sun: corona - stars: activity

\section{Introduction}

The nature of solar coronal bright points (BP) has been an enigma since their discovery in late 1960's (e.g. Vaiana et al. 1970). Their correspondence with small bipolar magnetic regions was found by combining ground-based magnetic field measurements with simultaneous space-borne X-ray imaging observations (Krieger et al. 1971; Golub et al. 1977). The bright points are clearly seen in $\mathrm{Ca} \mathrm{K}$ spectroheliograms as bright "knots" (e.g. Golub \& Pasachoff 1997). The daily number of BP's found on the Sun varies between several hundreds up to a few thousand (Golub et al. 1974). Zhang et al. (2001) found their density around 800 BP's for the entire solar surface at any moment.

Golub et al. (1974) found that the diameters of the bright points in X-rays are around $10^{4} \mathrm{~km}(10-20$ arcsec) and their lifetimes range from 2 hours to 2 days (see also Zhang et al. 2001). The physical studies have indicated the temperatures to be fairly low, $T \approx 2 \times 10^{6} \mathrm{~K}$, and the electron densities $n_{\mathrm{e}} \approx 5 \times$ $10^{9} \mathrm{~cm}^{-3}$ (e.g. Golub \& Pasachoff 1997), although cooler BP's exist (Habbal 1990). Assuming that almost all BP's represent new magnetic flux emerging at the solar surface, their overall

Send offprint requests to: $\mathrm{O}$. Vilh,

e-mail: osmi.vilhu@helsinki.fi

* Based on observations with the SUMER-spectrometer on board the $S O H O$ observatory.

$\star \star$ SUMER associated scientist.

$\star \star \star$ SUMER PI contribution to the solar magnetic flux would exceed that of the active regions (Golub \& Pasachoff 1997).

BP's often show irregular intensity and spatial shape variations on time scales of minutes which can be called as microflares (Shimojo \& Shibata 1999; Zhang et al. 2001). In particular, Shimojo and Shibata show that the frequency distribution of these microflares, as a function of the peak intensity, show a power law with index $1.7 \pm 0.4$ which is consistent with that of ordinary (stronger) flares. However, the total coronal heating can not energetically be due to these microflares, they are too few. Brown et al. (2001), using TRACE, studied for the first time BP's for their entire lifetime with a cadence of $2 \mathrm{~min}$ and spatial resolution of 0.5 arcsec, using hot FeXII and FeXI lines. In particular, they suggested that BP's are made up of a complex system of dense loops.

In the present paper we report observations of one particular bright point observed with the SUMER-spectrometer onboard $\mathrm{SOHO}$ using four ultraviolet lines formed between $2 \times 10^{4}-6 \times 10^{5} \mathrm{~K}$.

\section{Observations}

The observations were performed on October 4 and 8, 1996, with the SUMER normal incidence telescope of the $\mathrm{SOHO}$ observatory (Wilhelm et al. 1995). The full set of observations will be reported elsewhere (Huovelin et al. 2002); here we concentrate on a specific BP-complex observed October 8, 1996, between UT 15.20-17:20. 
Table 1. Lines observed. The formation temperatures assume ionisation equilibria from Arnaud \& Rothenflug (1985). The line intensities, widths $(F W H M)$ and non-thermal velocities $(\xi)$ represent measured mean values over the $8 \times 8$ arcsec area and over the 120 min observation around the bright point A in Fig. 2.

\begin{tabular}{cccccccc}
\hline \hline$\lambda(\mathrm{A})$ & Ion & lower level & upper level & temperature $(\mathrm{K})$ & int $\left(\mathrm{mW} / \mathrm{Sr} / \mathrm{m}^{2}\right)$ & $F W H M\left(\mathrm{~km} \mathrm{~s}^{-1}\right)$ & $\xi\left(\mathrm{km} \mathrm{s}^{-1}\right)$ \\
\hline 765.15 & NIV & $2 \mathrm{~s}^{2}{ }^{1} \mathrm{~S}_{0}$ & $2 \mathrm{~s} 2 \mathrm{p}^{1} \mathrm{P}_{1}$ & $10^{5}$ & $305 \pm 30$ & $91 \pm 5$ & $44 \pm 5$ \\
770.42 & NeVIII & $1 \mathrm{~s}^{2} 2 \mathrm{~s}^{2} \mathrm{~S}_{1 / 2}$ & $1 \mathrm{~s}^{2} 2 \mathrm{p}^{2} \mathrm{P}_{3 / 2}$ & $6 \times 10^{5}$ & $200 \pm 20$ & $91 \pm 5$ & $41 \pm 5$ \\
775.96 & NII & $2 \mathrm{~s}^{2} 2 \mathrm{p}^{2}{ }^{1} \mathrm{D}_{2}$ & $2 \mathrm{~s} 2 \mathrm{p}^{3}{ }^{1} \mathrm{D}_{2}$ & $2 \times 10^{4}$ & $22 \pm 5$ & $82 \pm 10$ & $40 \pm 10$ \\
787.74 & OIV & $2 \mathrm{~s}^{2} 2 \mathrm{p}^{2} \mathrm{P}_{1 / 2}$ & $2 \mathrm{~s} 2 \mathrm{p}^{2}{ }^{2} \mathrm{D}_{3 / 2}$ & $2 \times 10^{5}$ & $240 \pm 30$ & $95 \pm 5$ & $46 \pm 5$ \\
\hline
\end{tabular}

The region was found by first scanning a larger area near the disk center. The data consist of $23 \times 24$ raster scans of the detector $B$ first order. The scans were obtained by moving the spectrometer slit horizontally along the East-West (equatorial) direction in 2.25 arcsec steps, corresponding to $1700 \mathrm{~km}$ on the solar surface. The wavelength dispersion in each scan-point was perpendicular to the South-North direction, and 4 narrow spectral windows were observed simultaneously in each vertical spatial point (with 2 arcsec height). The observed wavelength intervals consist of 50 wavelength bins with a dispersion $0.0446-0.0447 \AA / \mathrm{px}\left(17 \mathrm{~km} \mathrm{~s}^{-1}\right.$, comparable to the thermal broadening of NIV 765 line formed at $10^{5} \mathrm{~K}$ ). The intervals were centered at the lines N IV (765.146 ̊), Ne VIII

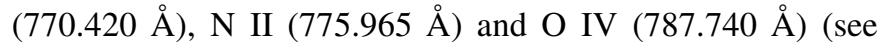
Table 1). The resolving power of SUMER within the observed wavelength range is close to $\lambda / \Delta \lambda=37000\left(8 \mathrm{~km} \mathrm{~s}^{-1}\right)$. For radial velocity measurements a better accuracy (around $4 \mathrm{~km} \mathrm{~s}^{-1}$ ) can be achieved. The scan time for each image was set to $2.8 \mathrm{~min}$ (6.5 $\mathrm{s}$ integration per slit position plus overheads), resulting in 44 images for each spectral line with spectral information inside each $2 \times 2.25$ arcsec spatial pixel.

The observations were reduced including flat-field and geometrical distortion corrections to the raw uncompressed FITS format data. These standard reductions were made with the SUMER reduction procedures written in IDL (Interactive Data Language, Registered Trademark) at the Institute of Theoretical Astrophysics, University of Oslo, Norway (M. Carlsson, private comm.). Dead time correction was not applied, since the total signal level in the slit was below $50000 \mathrm{cts} / \mathrm{s}$ (see the SUMER Data Cookbook, http://www.mpae.gwdg.de/ mpae_projects/ SUMER/text/cookbook . html).

The local gain correction was made to all spectra to compensate for gain variations. Finally, wavelength scaling and radiometric conversion to physical units were applied. For the two shortest wavelengths (765 and $770 \AA$ ) the scaling was $44.7 \mathrm{m \AA} / \mathrm{pixel}$, and for the other two, $44.6 \mathrm{~m} \AA / \mathrm{pixel}$. The radiometric calibration was based on the results reported by Wilhelm et al. (1997), the latest calibrations available in the WWWpages of SUMER during summer 1998 were used. The uncertainty in the absolute flux calibration is $\pm 15 \%$ (Schühle et al. 1998). The instrument compensated solar rotation during each sequence, and the spatial variability in each region could be monitored.

\section{Results}

Line profiles were fitted with the IDL Gaussfit-procedure, the mean values of resulting line parameters are given in Table 1.
These Gaussian fits resulted in line intensity, line center and line width $(F W H M)$ values at each spatial $(23 \times 24)$ and temporal (44, separated by $2.8 \mathrm{~min}$ ) points. Although the spectral resolution was $8 \mathrm{~km} \mathrm{~s}^{-1}$ and wavelength bins $17 \mathrm{~km} \mathrm{~s}^{-1}$, the fitting procedure of strong lines permits to measure the line centroids much better. Radial velocities and widths of the strongest lines can be determined with accuracy better than $5 \mathrm{~km} \mathrm{~s}^{-1}$ (see Fig. 6). Average non-thermal velocities are also included in Table 1 . They are slightly below the sonic velocities and are situated close to the upper boundary of the velocity - temperature correlation in quiet sun as derived by Dere \& Mason (1993, their Fig. 17).

Figure 1 shows the spectral windows observed. The quiet sun and spot spectra, as observed with the same instrument settings, can be found from the SUMER-atlas available via http://www.mpae.gwdg.de. Figure 2 shows the timeaveraged contour-plot of six observed frames (frames numbers 29-34 in Fig. 3). The observed region was extremely variable as shown in Fig. 3 where every time-frame is presented. Figure 4 shows the light curve of the NIV 765 line for the brightest and most persistent bright point in the observed region (A in Fig. 2) indicating strong mictoflaring. The variability is very coherent with strong correlation between individual $2 \times 2$ arcsec spatial pixels. If this bright point consisted of a complex loop system (not resolved with our spatial resolution but suggested by TRACE observations of Brown et al. 2001) this would mean a rapid avalance over the whole bright point region after triggering.

The line intensities were compared with those from the SUMER ATLAS (quiet sun and spot spectra) by dividing them with those of the quiet sun. The line ratios reflect relative emission measures and are proportional to $n_{\mathrm{e}}^{2} \times V$ where $n_{\mathrm{e}}$ is the electron density and $V$ the emitting volume at the line formation temperature (see Fig. 5). The background in the $50 \times 50$ arcsec region was somewhat different from the quiet network e.g. by having an excess hot component.

The observed region contained also some short lived transients, like BP-B close to the main BP-A (see Fig. 2). It showed clear $20 \mathrm{~km} \mathrm{~s}^{-1}$ up-flows during the outburst (see Fig. 6). On the other hand, BP-A did not show any variability in line shapes irrespective of it strong intensity variations.

\section{Discussion and conclusions}

The main bright point (A in Fig. 2 ) had dimensions $8 \times 8$ arcsec between $2 \times 10^{4}-6 \times 10^{5} \mathrm{~K}$. The non-thermal velocities (40-45 $\mathrm{km} \mathrm{s}^{-1}$ ) were somewhat smaller but close to the sonic ones. The most striking feature of the present observation, 

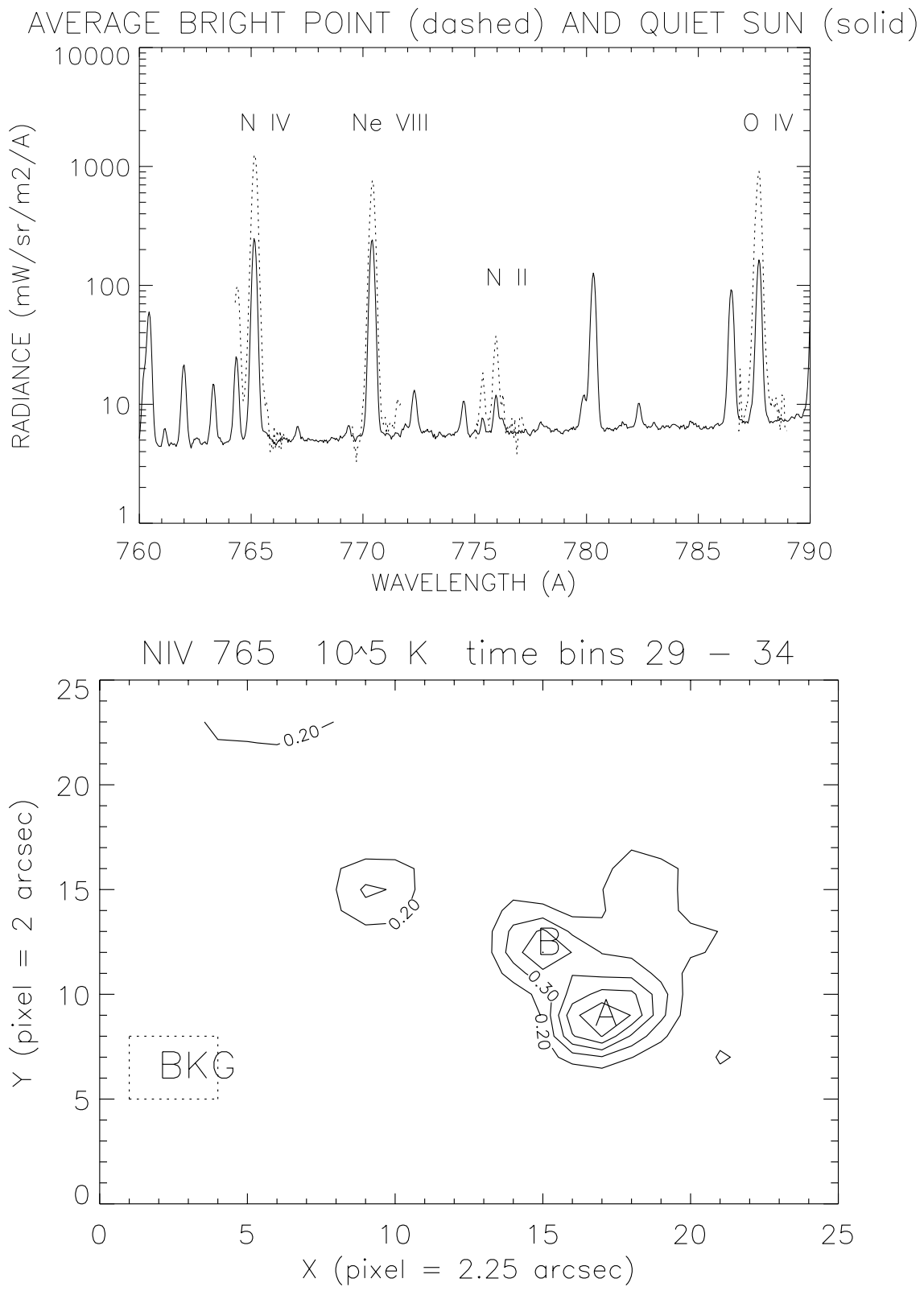

Fig. 1. The spectral range with the lines used marked. The average bright point spectra (dashed lines, BP-A in Fig. 2) are overplotted with the quiet sun spectrum from the SUMER-atlas (solid line, available via http://www . mpae. gwdg.de).
Fig. 2. NIV 765 contour-map $\left(\mathrm{W} / \mathrm{Sr} / \mathrm{m}^{2}\right)$ of the region observed. The map is an average of six time intervals (frames $29-34$ in Fig. 3). The bright points $\mathrm{A}$ and $\mathrm{B}$ discussed in the text are marked. "BKG" refers to the background region used in Figs. 4 and 5. The $X$-coordinate (in pixels, one pixel $=2.25$ arcsec) points to West and $Y$ (in pixels, one pixel $=2$ arcsec) to North. The map center is at 91 arcsec to West and 15 arcsec to South from the solar center in the beginning of observations. The region was observed in October 8, 1996, between UT 15:20-17:20 (two hours). lasting $120 \mathrm{~min}$, was the strong microflaring and the appearance of short lived transients. One of these (BP-B in Fig. 2) showed clear mass upflows with velocities $20 \mathrm{~km} \mathrm{~s}^{-1}$. No flows were detected elsewhere in the observed region.

Inside the $8 \times 8$ arcsec area of the BP-A no clear structure could be resolved within the 2 arcsec spatial resolution. All spatial pixels varied coherently with strong correlation between individual pixels (Fig. 4). It is possible that the BP-A consisted of a dense system of small loops triggering each others, so that the whole BP looked like a single variable object. A much better spatial resolution (like in TRACE, Brown et al. 2001) is needed to resolve the issue and to find the BP building blocks.

Comparing the sun and solar type active stars, Vilhu (1987, 1994) suggested a correlation between the CIV 1550 line intensity $\left(10^{5} \mathrm{~K}\right)$ and the magnetic filling factor. Lines formed at $10^{5} \mathrm{~K}$ arise close to the loop foot points and are good indicators of their surface area. In the quiet solar network the magnetic filling factor is at one per cent level (one per cent of the surface covered by equipartition fields) while in the most active (and consequently rapidly rotating) stars the filling factor approaches 100 per cent when the CIV line surface flux is 100 times that of the quiet sun. This suggests that the filling factor of BP-A was around 10 per cent (see Fig. 5). In this picture BP-A was similar to the average surface of a moderately active star (like solar type stars in the Hyades cluster, rotating with periods around 10 days or less). Further, some bright points (like the present one) may well be essentially like over dense quiet sun regions. However, much better spatial resolution will be needed to make any definite conclusion.

We also investigated the underlying magnetic field structure of the region during our observation (from the SOI-archive). The MDI-instrument onboard $\mathrm{SOHO}$ observed the region between UT 15:16-15:36 and 16:4517:05. In both MDI-observations (around the BP-A of Fig. 2) two bright positive flux regions (10 and 20 arcsec 


\section{NIV 0IV NeVIII NIV OIV NeVIII NIV 0IV NeVIII $\quad$ NIV 0IV NeVIII}

1
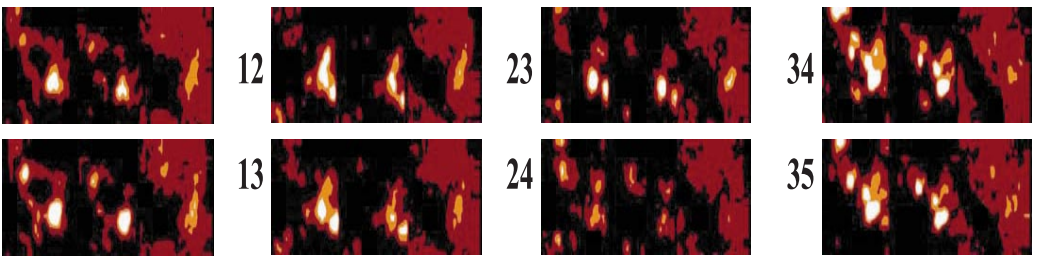

2
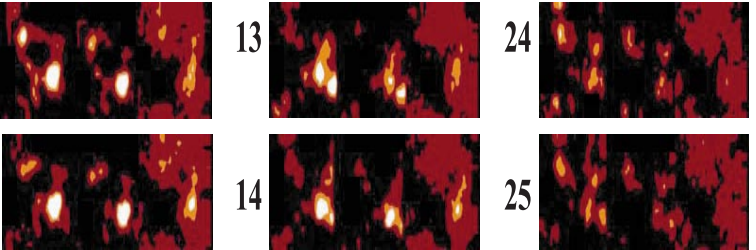

4
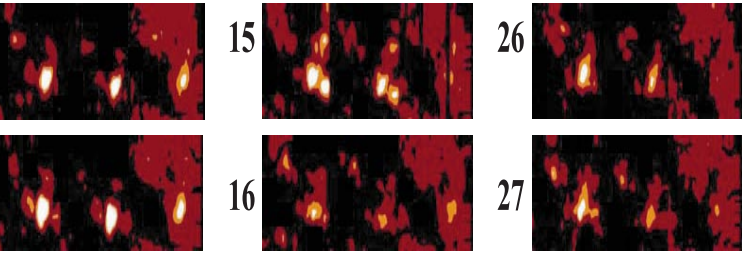

6
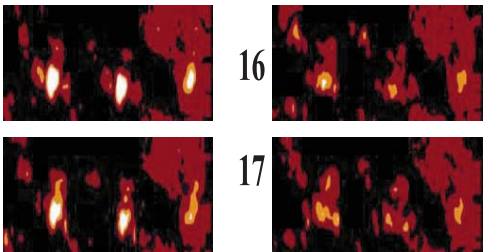

7

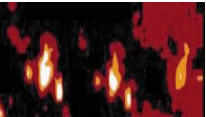

8

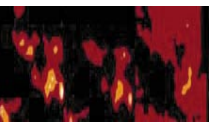

9

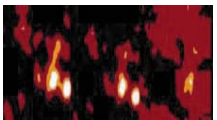

10
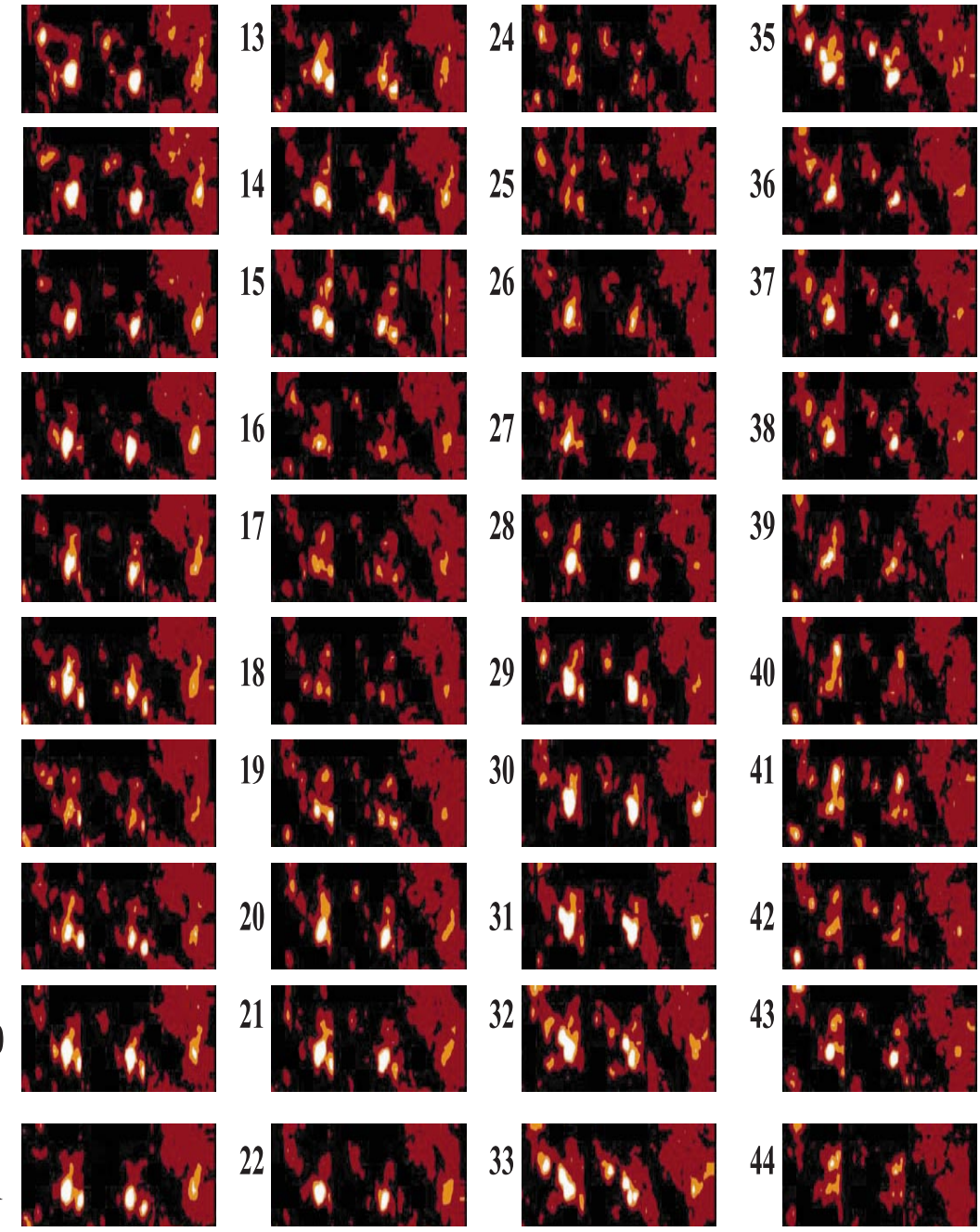

37
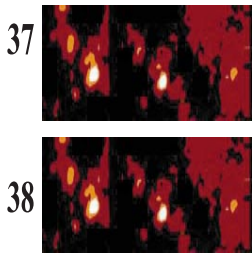

39

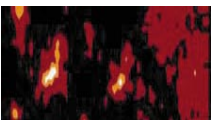

40
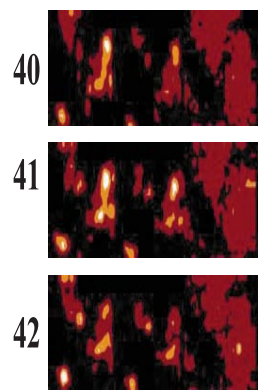

43

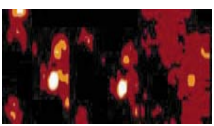

44

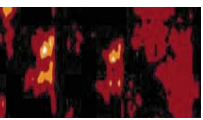

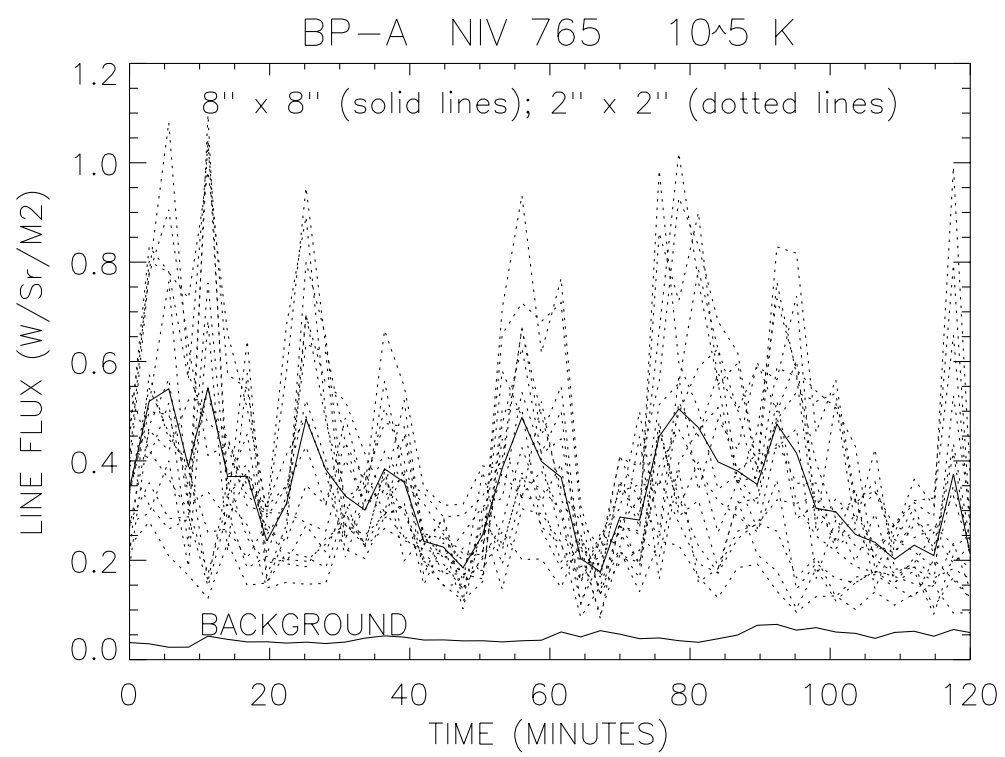

Fig. 4. The light curve of NIV 765 total intensity of the BP-A (see Fig. 2) using 16 spatial pixels $(2 \times 2$ arcsec each) and combining them into a single (average over $8 \times 8$ arcsec) light curve (the solid line). The light curves of all 16 individual spatial pixels are shown separately by dotted curves. Note the clear coherence of separate pixels. The background was computed from the $8 \times 8$ arcsec region shown in Fig. 2 as "BKG". 

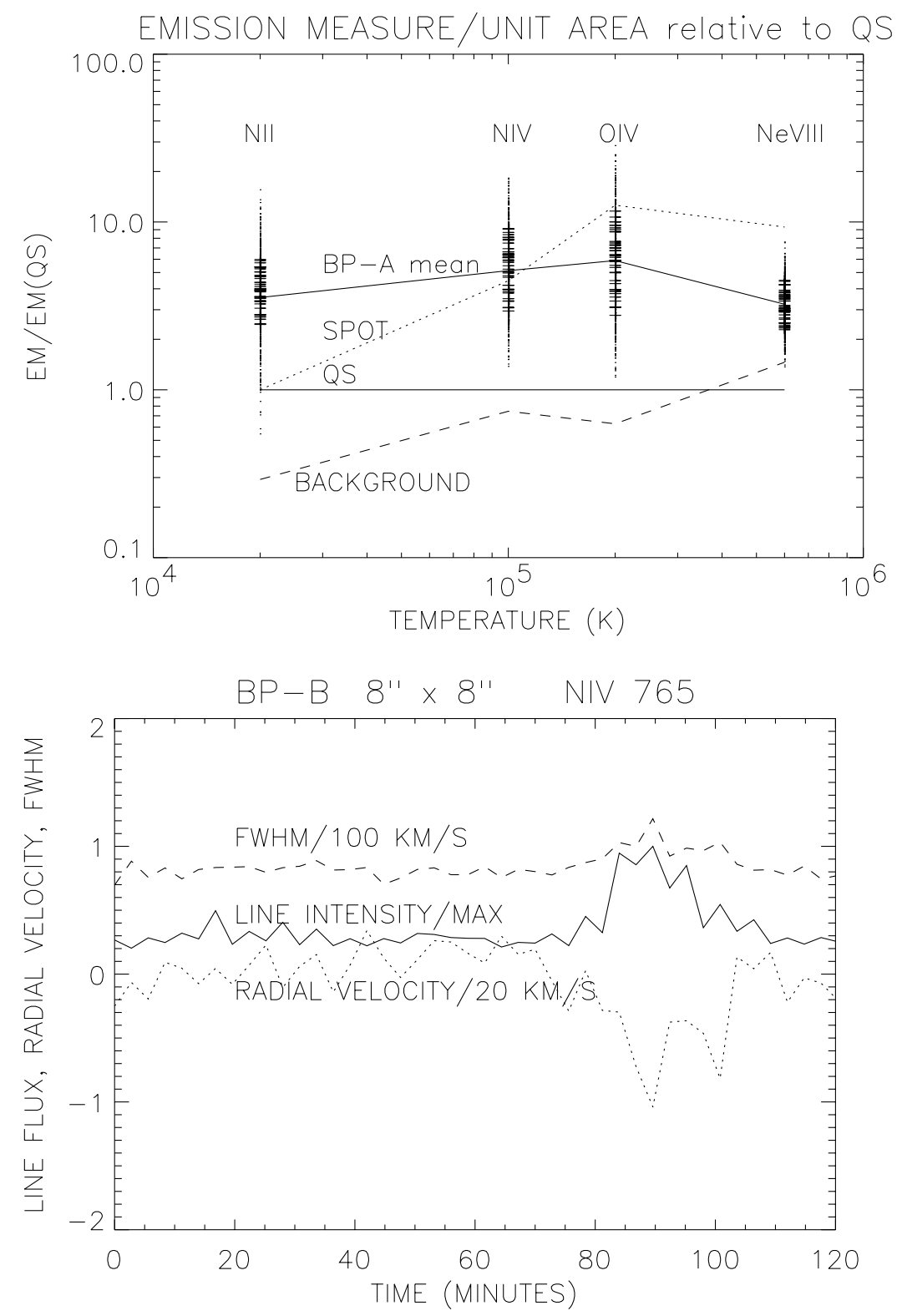

Fig. 5. Emission measures in the BP-A (see Fig. 2) vs. temperature, in units of the quiet sun value (from SUMER ATLAS, available via http://www.mpae.gwdg.de). These relative emission measures were computed simply as ratios of total line fluxes. They are proportional to $n_{\mathrm{e}}^{2} \times V$ where $n_{\mathrm{e}}$ is the electron density and $V$ the emitting volume at the formation temperature of the line in question. The short horizontal ticks give $8 \times 8$ arcsec averages for separate 44 time bins, while the dots (forming vertical lines) show all individual $2 \times 2$ arcsec pixels for all 44 time bins. The dashed line marked "BACKGROUND" was computed from the box shown in Fig. 2, while the dotted line shows the spot-spectrum from the SUMER ATLAS. It is suggested that $\mathrm{EM} / \mathrm{EM}(\mathrm{QS})$ at $10^{5} \mathrm{~K}$ is briefly equal to the magnetic flux tube filling factor (in per cent).

Fig. 6. Average (over $8 \times 8$ arcsec) light curves of the short lived transient B (see Fig. 2; appearing in frames numbers $29-34$ of Fig. 3) for the NIV-line total intensity, radial velocity (negative means blue-shifted upward motion) and line width $(F W H M)$. The average $F W H M$ is the same as that of the major bright point $\mathrm{A}$ (see Table 1) and indicates nonthermal broadening of $40 \mathrm{~km} \mathrm{~s}^{-1}$. This dynamic event may be associated magnetically with the neighbouring BP-A showing mass-upflows $20 \mathrm{~km} \mathrm{~s}^{-1}$. The errors of radial velocity and $F W H M$ are around $5 \mathrm{~km} \mathrm{~s}^{-1}$. apart) and two negative flux fragments (30 arcsec apart) are present and look rather persistent. According to Brown et al. (2001) one third of the bright points lie over emerging regions of magnetic flux while the remaining two thirds lie above cancelling magnetic features. It seems that the bright point studied in the present paper belongs to this latter category.

Acknowledgements. This work was performed with support from the Academy of Finland ( $\mathrm{OV}$ and $\mathrm{JH})$. We are very grateful to Matts Carlsson for providing software and useful information for basic data reductions. We thank the referee for valuable criticism.

\section{References}

Arnaud, M., \& Rothenflug, R. 1985, A\&AS, 60, 425

Brown, D. S., Parnell, C. E., Deluca, E. E., Golub, L., \& McMullen, R. A. 2001, Sol. Phys., 201, 305

Dere, K. P., \& Mason, E. 1993, Sol. Phys., 144, 217

Golub, L., Krieger, A. S., Silk, J. K., Timothy, A. F., \& Vaiana, G. S. 1974, ApJ, 189, L93
Golub, L., Krieger, A. S., Harvey, J. W., \& Vaiana, G. S. 1977, Sol. Phys., 53, 311

Golub, L., \& Pasachoff, J. M. 1997, The Solar Corona (Cambridge University Press, Cambridge, UK)

Habbal, S. R. 1990, in Mechanisms of Chromospheric and Coronal Heating, ed. P. Ulmschneider, E. R. Priest, \& R. Rosner (Springer Verlag), 127

Huovelin, J., Pohjolainen, S., Virtanen, J., Vilhu, O., \& Curdt, W. 2002, in preparation

Krieger, A. S., Vaiana, G. S., \& van Speybroeck, L. P. 1971, in Solar Magnetic Fields, ed. R. Howard, IAU Symp., 43, 397 (Reidel Publ. Co.)

Schühle, U., Brekke, B., Curdt, W., et al. 1998, Appl. Opt., 37, 2646 Shimojo, M., \& Shibata, K. 1999, ApJ, 516, 934

Vaiana, G. S., Krieger, A. S., van Speybroeck, L. P., \& Zehnpfennig, T. 1970, Bull. Am. Phys. Soc., 15, 611

Wilhelm, K., Curdt, W., Marsch, E., et al. 1995, Sol. Phys., 162, 189

Vilhu, O. 1987, in Cool Stars, Stellar Systems and the Sun, ed. J. L. Linsky, \& R. E. Stencel, Lecture Notes in Phys., 291, 110

Vilhu, O. 1994, Mem. S. A. It., 65, 61

Zhang, J., Kundu, M., \& White, S. M. 2001, Sol. Phys. 198, 347 